\title{
A NOVEL MULTISCALE THEORETICAL MODEL FOR DROPLET COALESCENCE IN TURBULENT DISPERSIONS
}

\author{
SHENGGAO GONG, LUCHANG HAN, HE'AN LUO \\ School of Chemical Engineering, Xiangtan University, Hunan, PR. China.
}

\begin{abstract}
A novel multi-scale theoretical model for coalescence rate of droplets induced by turbulence has been developed. This model proposed two kinds of interaction mechanisms between the underlying turbulent flow and droplets and can be a kernel function required for population balance model (PBM). Most previous models only considered the contribution of the eddies of size equal to the droplets to the coalescence since the velocity of droplet was assumed to be equal to that of eddies of same size; the sizes of droplets was usually assumed to fall in the inertial sub-range of turbulent energy spectrum and only considered the eddies in this sub-range to coalescence. But the distribution of the sizes of droplets may be quite wide in the device, considering the entire energy spectrum (i.e. containing the dissipation sub-range, the inertial sub-range and the energy-containing sub-range) may be more reasonable. The above assumptions in the previous models are no more needed in this work. A novel model based on the entire energy spectrum and the collisions between two droplets and between eddy and droplet has been derived. The contribution of eddies of different sizes to the coalescence has been considered. The results predicted by coupling novel model with PBM agree with experimental data.

Keywords: coalescence rate, droplet, entire energy spectrum, multiscale turbulent eddies, turbulence
\end{abstract}

\section{INTRODUCTION}

The droplet coalescence usually determines size distribution and interfacial area, and thus, has a significant effect on momentum, mass and energy transfer in turbulent multiphase flow. The size distributions and interfacial area can be predicted by coupling population balance model with coalescence model. However, the predicted results depend on the accuracy of coalescence model. Hence, it is meaningful to study droplet coalescence dynamics.

The coalescence of fluid particles (droplets or bubbles) due to turbulence is very complex. For simplification, the coalescence rate of fluid particles of sizes $d_{i}$ and $d_{j}, C\left(d_{i}, d_{j}\right)$, was usually estimated from the product of collision rate $\omega\left(d_{i}, d_{j}\right)$ and coalescence efficiency $P_{C}\left(d_{i}, d_{j}\right)$. $\omega\left(d_{i}, d_{j}\right)$ can be expressed as $(\pi / 4)\left(d_{i}+d_{j}\right)^{2} \bar{U}_{r} n_{i} n_{j}$. Here, $n_{i}$ and $n_{j}$ denote number density of fluid particles $i$ and $j$, respectively. The mean relative velocity of fluid particles, $\bar{U}_{r}$, is proportional to the square root of $\left(\bar{U}_{i}^{2}+\bar{U}_{j}^{2}\right)$. In the expression of $\omega\left(d_{i}, d_{j}\right)$, the mean velocity of fluid particle $d_{i}$ (e.g. $\bar{U}_{i}$ ) was usually taken as the mean velocity of eddies of size $d_{i}$ in inertial sub-range.

The following effects were not considered in above collision rate model. (1) Eddies of sizes larger than the longitudinal integral length scale $L$ may not be able to effectively move fluid particles [1]. (2) The reduction of free space for fluid particle movement due to the existence of dispersed phase [2-4]. (3) The collision events should not be counted when the distance between fluid particles is larger than the moving distance of a fluid particle due to turbulence [3, 4]. (4) Contribution of eddies in the entire energy spectrum to fluid particle coalescence should be considered since the sizes of fluid particles may not always fall in the inertial sub-range [5, 6]. Many collision rate models were proposed to consider above four aspects. However, the mean velocity of fluid particles in these models is still assumed to be equal to the velocity of eddies of the length scale of fluid particles. 
The other important part of coalescence rate model is coalescence efficiency. To our knowledge, three kinds of coalescence efficiency model were presented in the literature. That is, the energy, the critical approach velocity and the film-drainage models. The energy and the critical approach velocity models were estimated on the basis of experimental results, and the application scope of them will be restricted by the experimental conditions. The film-drainage model is associated with contact time and drainage time between fluid particles. Since it was proposed by Coulaloglou and Tavlarides [7], the film-drainage model has received wide attention. Many works focused on the contact time and the drainage time, and many expressions have been proposed to estimate those two time scales [8-10].

The above review showed that $\bar{U}_{i}$ was usually taken as the mean velocity of eddies of size $d_{i}$. It may imply that these coalescence models only considered the contribution of eddies of sizes equal to the fluid particle size. Since the motion of droplet $d_{i}$ may be driven by eddies of various sizes, the collision between droplets could be caused by both small eddies (less than and equal to the size of droplets) and large eddies (larger than the size of droplets). Therefore, the contribution of eddies of various sizes to the coalescence of droplets of given sizes should be considered. Furthermore, the interactions between eddies and droplets are the prerequisite for the collision between droplets, but it was not reflected in the previous models. Finally most of existing coalescence models only considered the contribution of eddies in inertial sub-range, and the droplet coalescence should be simulated in the entire energy spectrum since the droplet size may not always fall in inertial sub-range. Hence, considering the above aspects, a novel coalescence model will be developed in this work.

\section{MODEL DEVELOPMENT}

This work aims to study the droplet coalescence caused by turbulence. Two different mechanisms, which can lead to the coalescence, are proposed (please see Fig. 1).

For the double-eddy mechanism depicted in Fig. 1, the collision between droplets $d_{i}$ and $d_{j}$ is caused by eddies $\lambda_{i}$ and $\lambda_{j}$. Three events contribute to the final collision between droplets in double-eddy mechanism. On the one hand eddy $\lambda_{i}$ interacts with droplet $d_{i}$ (i.e. event $A$ ), and on the other hand eddy $\lambda_{j}$ interacts with droplet $d_{j}$ (i.e. event $B$ ), then droplet $d_{i}$ and droplet $d_{j}$ collide with each other at a certain velocity (i.e. event $C$ ) after the two droplets obtain the kinetic energy from these eddies. Note that, the volume of large eddy will expand if the droplet is entrained by the eddy.

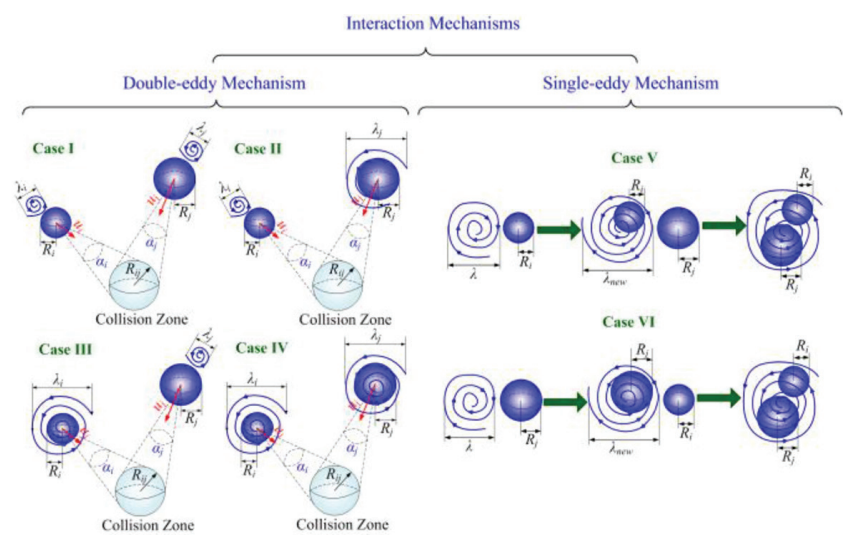

Figure 1: Two different interaction mechanisms between eddies and droplets. 
The collision between droplets $d_{i}$ and $d_{j}$ is caused by an eddy of size $\lambda$ in single-eddy mechanism. Take the case V in Fig. 1 as an example, and the collision between droplets in single-eddy mechanism also includes three events. Firstly eddy $\lambda$ interacts with droplet $d_{i}$ (i.e. event $A$ ), meanwhile the size of eddy $\lambda$ will become $\lambda_{\text {new }}$ defined by $\left(d_{i}^{3}+\lambda_{i}^{3}\right)^{1 / 3}$ after droplet $d_{i}$ is entrained by eddy $\lambda$. Then eddy $\lambda_{\text {new }}$ interacts with droplet $d_{j}$ (i.e. event $B$ ). Finally the collision between droplets occurs after eddies transfer their energy to droplets (i.e. event $C$ ).

According to above description, events $A$ and $B$ are independent of each other, and the collision/coalescence rate between droplets essentially depends on the probability of occurrence of the event $C, P(C)$. Set $D$ is defined here to determine $P(C)$. Set $D$ represents the set of collisions between droplet $d_{i}$ and droplet $d_{j}$ with arbitrary velocities, and the probability of occurrence of set $D$ is $P(D)$. Obviously, event $C$ is one of events in set $D$. The probabilities of occurrence of events $A$ and $B$ are $P(A)$ and $P(B)$, respectively. The joint probability of occurrence of events $A$ and $B, P(A \cap B)$, could be expressed as the product of $P(A)$ and $P(B)$. Since event $C$ is induced by events $A$ and $B$, the probability of occurrence of event $C$ in the set $D$ can be expressed as the product of $P(A \cap B)$ and $P(D)$. That is, $P(C)=P(A) P(B) P(D)$.

Following above method, the collision probability between droplets (i.e. $P(D)$ ) as well as the collision probability between eddies and droplets (i.e. $P(A)$ or $P(B)$ ) should be given first. Then, collision rate between droplets can be obtained by using the collision probability between droplets caused by eddies of given sizes (i.e. $P(C)$ ). Finally, the coalescence rate between droplets can be determined by combining collision rate and coalescence efficiency.

\subsection{Collision Probability between Droplets}

The collision process between two nano-particles was studied by Peev [11], and collision probability of two nano-particles was also obtained according to the definition of solid angle. The collision between droplets can be analogous to the collision between nano-particles. Following Peev's method, when the initial distance between the surfaces of droplets in the direction of the connecting line of two mass centers is $s$, the collision probability between droplets of sizes $d_{i}$ and $d_{j}$ could be expressed as

$$
P\left(s, d_{i}, d_{j}\right)= \begin{cases}{\left[\left(s+R_{i j}\right)-\left(s^{2}+2 s R_{i j}\right)^{1 / 2}\right] /\left[2\left(s+R_{i j}\right)\right]} & \text { For } 0 \leq s \leq s^{*} \\ {\left[R_{i j}^{2}-\left(s+R_{i j}-L_{d_{j} \rightarrow d_{i}}\right)^{2}\right] /\left[4 L_{d_{j} \rightarrow d_{i}}\left(s+R_{i j}\right)\right]} & \text { For } s^{*} \leq s \leq L_{d_{j} \rightarrow d_{i}}\end{cases}
$$

where, $P\left(s, d_{i}, d_{j}\right)$ denotes collision probability of droplets $d_{i}$ and $d_{j}$ at an initial distance of $s$ ranging from 0 to $L_{d_{j} \rightarrow d_{i}} \cdot R_{i j}=\left(d_{i}+d_{j}\right) / 2 \cdot s^{*}=\left(L_{d_{j} \rightarrow d_{i}}^{2}+R_{i j}^{2}\right)^{1 / 2}-R_{i j} \cdot L_{d_{j} \rightarrow d_{i}}$ is the mean collision free path of droplets.

\subsection{Collision Probability between Eddies and Droplets}

The collision between eddies and droplets occurs by a mechanism analogous to droplet collisions. Thus the collision probability between eddies and droplets in the range of $\left[0, L_{\lambda \rightarrow d}\right]$ could be described by eqn (1). Here, $L_{\lambda \rightarrow d}$ is the mean collision free path between eddy $\lambda$ and droplet $d$. However, unlike the collision between droplets, the collision between eddies and droplets will be restricted by the life time $\tau_{e}$ of eddies. The longest travelling distance of eddies during their life time could be approximated as $\bar{u}_{\lambda} \tau_{e}$, Hence, when eqn (1) is used to estimate the collision probability between eddies and droplets, the mean collision free path should be modified as $L_{\lambda \rightarrow d}^{*}$ (i.e. $\left.\min \left(\bar{u}_{\lambda} \tau_{e}, L_{\lambda \rightarrow d}\right)\right)$. In addition, eddies may be generated 
before the formation of droplets, it may lead to that eddies couldn't collide with droplets when $s_{\lambda, d} \leq \bar{u}_{\lambda} \tau_{e}$. Since the generation process is random, for these eddies that are generated before the formation of droplets, this work considers the collision of these eddies with droplets is fully random. Therefore, the collision probabilities between the droplets and eddies that are generated before the formation of droplets are 1/2. Finally, the collision probability, $P\left(s_{\lambda, d}, \lambda, d\right)$, between eddies and droplets could be obtained by considering the above two aspects on the basis of eqn (1). Then,

$$
\begin{aligned}
& P\left(s_{\lambda, d}, \lambda, d\right)=
\end{aligned}
$$

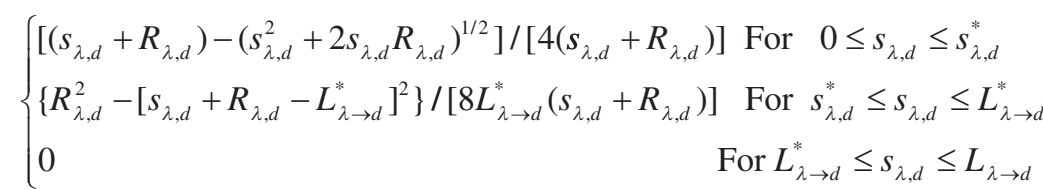

where, $P\left(s_{\lambda, d}, \lambda, d\right)$ denotes the collision probability between eddy $\lambda$ and droplet $d$ at an initial distance of $s_{\lambda, d}$ ranging from 0 to $L_{\lambda \rightarrow d^{*}} s_{\lambda, d}^{*}=\left[\left(L_{\lambda \rightarrow d}^{*}\right)^{2}+R_{\lambda, d}^{2}\right]^{1 / 2}-R_{\lambda, d}$ and $R_{\lambda, d}=(\lambda+d) / 2$.

As seen from eqn (2), $P\left(s_{\lambda, d}, \lambda, d\right)$ is related with $s_{\lambda, d}$, which is difficult to estimate from theory. Since eddies are randomly generated, $s_{\lambda, d}$ could be considered as independent random variable and follows random distribution. This work considers the value of $s_{\lambda, d}$ to be evenly distributed within the range of $\left[0, L_{\lambda \rightarrow d}\right]$. Then the mean collision probability $P(\lambda, d)$ can be obtained by integrating $s_{\lambda, d}$ in the range of $\left[0, L_{\lambda \rightarrow d}\right] . P(\lambda, d)$ is used in this work to represent the collision probability between droplets and eddies.

$$
P(\lambda, d)=\int_{0}^{L_{\lambda \rightarrow d}} P\left(s_{\lambda, d}, \lambda, d\right) d s_{\lambda, d} / L_{\lambda \rightarrow d}
$$

Note that, the sizes $\lambda$ and $d$ are general variables in eqn (3), and they will be replaced by different eddy sizes and different droplet sizes in the following.

\subsection{Coalescence Rate Model}

In the literature, the coalescence rate between droplets is generally represented by the product of collision rate and coalescence efficiency. In this section, according to the collision probabilities in the sections 2.1 and 2.2, the collision rate will be determined first.

\subsubsection{Collision Rate}

Following above method, for double-eddy mechanism, the collision probability between droplet $d_{i}$ and droplet $d_{j}$ caused by eddies $\lambda_{i}$ and $\lambda_{j}, P_{D E M}$, can be expressed as

$$
P_{D E M}=P\left(\lambda_{i}, d_{i}\right) P\left(\lambda_{j}, d_{j}\right) P\left(s, d_{i}, d_{j}\right)
$$

Similarly, for single-eddy mechanism, the collision probability between droplet $d_{i}$ and droplet $d_{j}$ caused by a single eddy $\lambda, P_{S E M}$, can be also expressed as

$$
P_{S E M}=P\left(\lambda, d_{i}\right) P\left(\lambda_{\text {new }}, d_{j}\right) P\left(s, d_{i}, d_{j}\right)
$$

Take the centroid of droplet $d_{i}$ as center, two spheres with the radii $\left(s+R_{i j}\right)$ and $\left(s+R_{i j}+\mathrm{d} s\right)$, respectively, are considered. The number of droplets $\mathrm{d} n_{j}$ of size $d_{j}$ enclosed between these spheres is given by

$$
\mathrm{d} n_{j}=n_{j} \mathrm{~d} V=4 \pi n_{j}\left(s+R_{i j}\right)^{2} \mathrm{~d} s
$$


where $\mathrm{d} s$ is a very small quantity and all droplets $d_{j}$ from eqn (6) have the same solid angle and the same probability for the collision with droplet $d_{i}$. The number $\mathrm{d} N_{j}$ of droplet $d_{j}$ that can collide with droplet $d_{i}$ in the volume $\mathrm{d} V$ is

$$
\mathrm{d} N_{j}=\left\{\begin{array}{l}
P_{D E M} \mathrm{~d} n_{j} \\
P_{S E M} \mathrm{~d} n_{j}
\end{array}=4 \pi n_{j}\left(s+R_{i j}\right)^{2} \mathrm{~d} s \times \begin{cases}P_{D E M} & \text { For double-eddy mechanism } \\
P_{S E M} & \text { For single-eddy mechanism }\end{cases}\right.
$$

Probability $P_{D E M}$ or $P_{S E D}$ depends on the initial distance $s$. Integrating eqn (7) with respect to $s$ from 0 to $L_{d_{j} \rightarrow d_{i}}$, for two different mechanisms the number of droplet $d_{j}$ that can collide with droplet $d_{i}, N_{j}$, can be represented by eqns. (8) and (9), respectively.

$$
\begin{gathered}
N_{j}=\int_{n}^{L_{d_{j} \rightarrow d_{i}}} \mathrm{~d} N_{j}=4 \pi n_{j}\left(d_{i}+d_{j}\right)^{2} L_{d_{i} \rightarrow d_{i}} P\left(d_{i}, \lambda_{i}\right) P\left(d_{j}, \lambda_{j}\right) \\
N_{j}=\int_{n}^{L_{d_{j} \rightarrow d_{i}}} \mathrm{~d} N_{j}=4 \pi n_{j}\left(d_{i}+d_{j}\right)^{2} L_{d_{i} \rightarrow d_{i}} P\left(d_{i}, \lambda\right) P\left(d_{j}, \lambda_{\text {new }}\right)
\end{gathered}
$$

For the collision between two droplets, we might as well to assume that the droplet $d_{i}$ is immobile and the droplet $d_{j}$ is mobile with an average relative velocity $\bar{U}_{r}$. When the value of the initial distance between droplets $d_{j}$ and $d_{i}$ tends to 0 , droplet $d_{j}$ will collide with droplet $d_{i}$ in a very short time interval. However, if the initial distance equals to $L_{d_{i} \rightarrow d_{i}}$, the two droplets will collide after the time $\mathrm{d} t: \mathrm{d} t=L_{d_{j} \rightarrow d_{i}} / \bar{U}_{r}$. Hence, the droplet $d_{i}$ will have $N_{j}$ collisions with droplet $d_{j}$ during the time interval $\mathrm{d} t$, and the number of collisions of droplet $d_{i}$ with the set of droplet $d_{j}$ per unit time can be written as

$$
\Omega\left(d_{i}, d_{j}\right)=N_{j} / \mathrm{d} t=N_{j} \bar{U}_{r} / L_{d_{j} \rightarrow d_{i}}
$$

Droplet $d_{i}$ is an arbitrary droplet of size $d_{i}$, and each droplet $d_{i}$ will have $\Omega\left(d_{i}, d_{j}\right)$ collisions with droplet $d_{j}$. Therefore, the number of collisions between droplets $d_{i}$ and $d_{j}$ per unit physical space volume per unit time (also called collision rate), $\omega\left(d_{i}, d_{j}\right)$, can be determined as

$$
\omega\left(d_{i}, d_{j}\right)=\Omega\left(d_{i}, d_{j}\right) n_{i}
$$

\subsubsection{Coalescence Efficiency}

It is generally accepted that the coalescence will occur for a collision between droplets if the contact time $\tau_{c}$ is larger than the drainage time $\tau_{d}$. The ratio of $\tau_{d} / \tau_{c}$ can thus provide a first indication of whether coalescence will occur. The following function has been widely used to calculate coalescence efficiency:

$$
P_{C}\left(d_{i}, d_{j}\right)=\exp \left(-\tau_{d} / \tau_{c}\right)
$$

Many expressions or models for $\tau_{c}$ and $\tau_{d}$ are presented in the literature. The model of Luo [10] is used in this work and can be written as

$$
P_{C}\left(d_{i}, d_{j}\right)=\exp \left[-c_{l s} \frac{\sqrt{0.75\left(1+\xi^{2}\right)\left(1+\xi^{3}\right)}}{\left(\rho_{d} / \rho_{c}+C_{V M}\right)(1+\xi)^{3}} \sqrt{\frac{\rho_{c} d_{i} \bar{U}_{r}^{2}}{\sigma}}\right]
$$

where $\rho_{c}$ and $\rho_{d}$ are densities of continuous and dispersed phases, respectively; $\sigma$ is the surface tension. $\xi=d_{i} / d_{j}$. The coefficient of virtual mass $C_{V M}$ is calculated by the expression of Kamp et al. [8], and the model parameter $c_{l s}$ is taken as 1.0 in this work. 


\subsubsection{Coalescence Rate}

The coalescence rate between droplets caused by eddies could be expressed as the product of eqns (11) and (13). When considering the contribution of eddies in the entire energy spectrum to the coalescence of droplets of given sizes, eddies of various sizes may have different contributions to the coalescence since the collisions between droplets are associated with the interactions between eddies and droplets. The difference of contribution of eddies with different sizes to the coalescence needs to be considered. The number density of eddies varies with their sizes. Hence, a probability density function for the number density of eddies in the entire energy spectrum is introduced to consider this difference and could be expressed as

$$
f_{n_{\lambda}}=n_{\lambda} / N_{\lambda, t o t}=n_{\lambda} / \int_{\lambda_{\min }}^{\lambda_{\max }} n_{\lambda} d \lambda
$$

Based on eqns (11), (13) and (14), for two different mechanisms, the total coalescence rate between droplet $d_{i}$ and droplet $d_{j}$ caused by eddies could be obtained by taking into account all possible values of sizes of eddies in the entire energy spectrum.

For double-eddy mechanism, the total coalescence rate $C_{t o t, D E M}\left(d_{i}, d_{j}\right)$ could be determined by integrating with respect to $\lambda_{i}$ and $\lambda_{j}$ from $\lambda_{\min }$ to $\lambda_{\max }$. Since the exchangeability of the size groups of $\left[\lambda_{i}, d_{i}\right]$ and $\left[\lambda_{j}, d_{j}\right]$, the repeated coalescence events should be removed in double-eddy mechanism. In the double-eddy mechanism, the integral interval is divided into four parts to remove repeated events. The four sub integral intervals correspond to the cases of I IV in Fig. 1. If $d_{i} \leq d_{j}$, the integral interval for each case can be given as: $\lambda_{\min } \leq \lambda_{i} \leq d_{i} \& \lambda_{\text {min }}$ $\leq \lambda_{j} \leq d_{j}$ for case I; $\lambda_{\text {min }} \leq \lambda_{i} \leq d_{i} \& d_{j}<\lambda_{j} \leq \lambda_{\text {max }}$ for case II; $d_{i}<\lambda_{i} \leq \lambda_{\text {max }} \& \lambda_{\text {min }} \leq \lambda_{j} \leq d_{j}$ for case III; $d_{i}<\lambda_{i} \leq \lambda_{\text {max }} \& d_{j}<\lambda_{j} \leq \lambda_{\text {max }}$ for case IV. According to the integral properties, the total coalescence rate for double-eddy mechanism can be expressed as the sum of the total coalescence rates of the cases of $\mathrm{I} \sim \mathrm{IV}$, that is

$$
\begin{aligned}
& C_{t o t, D E M}\left(d_{i}, d_{j}\right)=C_{t o t}^{I}\left(d_{i}, d_{j}\right)+C_{t o t}^{I I}\left(d_{i}, d_{j}\right)+C_{t o t}^{I I I}\left(d_{i}, d_{j}\right)+C_{t o t}^{I V}\left(d_{i}, d_{j}\right) \\
& C_{t o t}^{I}\left(d_{i}, d_{j}\right)=\frac{1}{\gamma_{1}} \int_{\lambda_{\min }}^{d_{i}} \int_{\lambda_{\min }}^{d_{i}} f\left(\lambda_{i}, \lambda_{j}, d_{i}, d_{j}\right) d \lambda_{j} d \lambda_{i}+\frac{1}{\gamma_{2}} \int_{\lambda_{\min }}^{d_{i}} \int_{d_{i}}^{d_{j}} f\left(\lambda_{i}, \lambda_{j}, d_{i}, d_{j}\right) d \lambda_{j} d \lambda_{i} \\
& C_{t o t}^{I I}\left(d_{i}, d_{j}\right)=\frac{1}{\gamma_{2}} \int_{\lambda_{\min }}^{d_{i}} \int_{d_{j}}^{\lambda_{\max }} f\left(\lambda_{i}, \lambda_{j}, d_{i}, d_{j}\right) d \lambda_{j} d \lambda_{i} \\
& C_{\text {tot }}^{I I I}\left(d_{i}, d_{j}\right)=\frac{1}{\gamma_{2}} \int_{d_{i}}^{\lambda_{\max }} \int_{\lambda_{\min }}^{d_{i}} f\left(\lambda_{i}, \lambda_{j}, d_{i}, d_{j}\right) d \lambda_{j} d \lambda_{i}+\frac{1}{\gamma_{1}} \int_{d_{i}}^{d_{j}} \int_{d_{i}}^{d_{j}} f\left(\lambda_{i}, \lambda_{j}, d_{i}, d_{j}\right) d \lambda_{j} d \lambda_{i} \\
& +\frac{1}{\gamma_{2}} \int_{d_{j}}^{\lambda_{\max }} \int_{d_{i}}^{d_{j}} f\left(\lambda_{i}, \lambda_{j}, d_{i}, d_{j}\right) d \lambda_{j} d \lambda_{i} \\
& C_{\text {tot }}^{I V}\left(d_{i}, d_{j}\right)=\frac{1}{\gamma_{2}} \int_{d_{i}}^{d_{j}} \int_{d_{j}}^{\lambda_{\max }} f\left(\lambda_{i}, \lambda_{j}, d_{i}, d_{j}\right) d \lambda_{j} d \lambda_{i}+\frac{1}{\gamma_{1}} \int_{d_{j}}^{\lambda_{\max }} \int_{d_{j}}^{\lambda_{\max }} f\left(\lambda_{i}, \lambda_{j}, d_{i}, d_{j}\right) d \lambda_{j} d \lambda_{i} \\
& f\left(\lambda_{i}, \lambda_{j}, d_{i}, d_{j}\right)=\omega\left(d_{i}, d_{j}\right) P_{C}\left(d_{i}, d_{j}\right) f_{n_{\lambda_{i}}} f_{n_{\lambda_{j}}}
\end{aligned}
$$

Here, $C_{t o t}^{I}\left(d_{i}, d_{j}\right), C_{t o t}^{I I}\left(d_{i}, d_{j}\right), C_{t o t}^{I I I}\left(d_{i}, d_{j}\right)$ and $C_{t o t}^{I V}\left(d_{i}, d_{j}\right)$ denote the total coalescence rates of cases of I IV in Fig. 1 , respectively. The factor $\gamma_{1}$ will be taken as 2 when $d_{i}=d_{j} \& \lambda_{i} \neq \lambda_{j}$ due to the exchangeability of the sizes $\lambda_{i}$ and $\lambda_{j}$, otherwise $\gamma_{1}$ will be taken as $1 . \gamma_{2}$ will be always taken as 1 since the sizes $\lambda_{i}$ and $\lambda_{j}$ are non-exchangeable. Note that, the cases of II and III in 
Fig. 1 are equivalent due to the exchangeability of the size groups of $\left[\lambda_{i}, d_{i}\right]$ and $\left[\lambda_{j}, d_{j}\right]$ when $d_{i}=d_{j}$.

Since the difference of interactions between droplets of various sizes and eddies, the single-eddy mechanism contains two different collision cases (i.e. cases V and VI in Fig. 1). However, these two cases cannot coexist simultaneously. For single-eddy mechanism, the total coalescence rate $C_{\text {tot,SEM }}\left(d_{i}, d_{j}\right)$ can be expressed as

$$
\text { or } \begin{aligned}
C_{t o t, S E M}\left(d_{i}, d_{j}\right) & =C_{t o t}^{V}\left(d_{i}, d_{j}\right) \\
C_{t o t, S E M}\left(d_{i}, d_{j}\right) & =C_{t o t}^{V I}\left(d_{i}, d_{j}\right)
\end{aligned}
$$

Here, $C_{t o t}^{V}\left(d_{i}, d_{j}\right)$ and $C_{t o t}^{V I}\left(d_{i}, d_{j}\right)$ denote the total coalescence rates of cases of V and VI in Fig. 1, respectively.

When the coalescence is caused by a single eddy, the necessary condition that two droplets can be entrained by an eddy is $\lambda \geq d_{i}$ or $d_{j}$ and $\lambda_{\text {new }} \geq\left(d_{i}+d_{j}\right)$. Thus, the integral lower limits for the cases V and VI could be taken as $\max \left[\left(d_{i}^{3}+d_{j}^{3}\right)^{1 / 3}-d_{i}, d_{i}\right]$ and $\max \left[\left(d_{i}^{3}+d_{j}^{3}\right)^{1 / 3}\right.$ $\left.-d_{j}, d_{j}\right]$, respectively. The coalescence rates for the cases V and VI can be written as eqns (22) and (23), respectively. Especially, the cases of V and VI are equivalent for equal-sized droplets.

$$
\begin{aligned}
& C_{t o t, V}\left(d_{i}, d_{j}\right)=\int_{\max \left[\left(d_{i}^{3}+d_{j}^{3}\right)^{1 / 3}-d_{i}, d_{i}\right]}^{\lambda_{\max }} \omega\left(d_{i}, d_{j}\right) P_{C}\left(d_{i}, d_{j}\right) f_{n_{\lambda}} d \lambda \\
& C_{t o t, V I}\left(d_{i}, d_{j}\right)=\int_{\max \left[\left(d_{j}^{3}+d_{i}^{3}\right)^{1 / 3}-d_{j}, d_{j}\right]}^{\lambda_{\max }} \omega\left(d_{i}, d_{j}\right) P_{C}\left(d_{i}, d_{j}\right) f_{n_{\lambda}} d \lambda
\end{aligned}
$$

Finally, the total coalescence rate of droplets caused by eddies could be written as

$$
C_{t o t}\left(d_{i}, d_{j}\right)=C_{t o t, D E M}\left(d_{i}, d_{j}\right)+C_{t o t, S E M}\left(d_{i}, d_{j}\right)
$$

\subsection{Modeling Closure}

To consider the coalescence in the entire energy spectrum, the turbulent parameters such as energy spectrum function $E(\kappa)$, mean kinetic energy of eddies $\bar{e}(\lambda)$ and number density of eddies $n_{\lambda}$ should be given first. In addition, the unknown parameters, such as the mean relative velocity $\bar{U}_{r}$ and the mean collision free path (e.g. $L_{\lambda \rightarrow d}$ ) need to be determined to close the model. All these parameters related with coalescence model are given following.

\subsubsection{Turbulent Parameters}

Only two energy spectrum functions could be found from the literature to cover all the turbulent energy range and they were proposed by Pope [12] and Hinze [13], respectively. Han et al. [5] found that the Pope's function is more reasonable than the Hinze's function. Therefore, the Pope's function is used to model coalescence.

If the turbulence could be regarded as a series of eddies with certain volume, the mean kinetic energy of eddies of size $\lambda$ could be expressed as $\pi \lambda^{3} \rho_{c} \bar{u}_{\lambda}^{2} / 12$. The mean square velocity, $\bar{u}_{\lambda}^{2}$, could be roughly estimated by the second-order longitudinal structure function $\left\langle[\Delta u]^{2}\right\rangle(\lambda)$ valid for entire energy spectrum proposed by Sawford and Hunt [14]. Based on the definition of energy spectrum, the number density of eddies of size $\lambda$ can be determined [15]. 


\subsubsection{Mean Relative Velocity}

According to direct numerical simulation, the probability density function (PDF) of velocities in isotropic turbulence closely follows Gaussian distribution. In addition, kinetic energy of droplets is obtained from turbulence. Therefore, this work argues that the velocity PDF of droplets of various sizes caused by eddies of size $\lambda$ follows Gaussian distribution. Since the turbulence is random, the motion of droplets could be considered as independent of each other. Thus, the joint PDF $f\left(\vec{U}_{i}, \vec{U}_{j}\right)$ of velocities of two droplets can be expressed as

$$
f\left(\vec{U}_{i}, \vec{U}_{j}\right)=\frac{1}{\left(2 \pi \sigma_{U_{i}}^{2}\right)^{3 / 2}} \exp \left[-U_{i}^{2} /\left(2 \sigma_{U_{i}}^{2}\right)\right] \frac{1}{\left(2 \pi \sigma_{U_{j}}^{2}\right)^{3 / 2}} \exp \left[-U_{j}^{2} /\left(2 \sigma_{U_{j}}^{2}\right)\right]
$$

$\sigma_{U_{i}}^{2}$ and $\sigma_{U_{j}}^{2}$ are the variances for velocity PDF of droplets of sizes $d_{i}$ and $d_{j}$, respectively.

Equation (25) includes variables $\vec{U}_{i}$ and $\vec{U}_{j}$, but we focus on the relative velocity $\vec{U}_{r}$ of two droplets. By using the transformation relationship between $\vec{U}_{i}$ and $\vec{U}_{r}$ proposed by Das [16], $\bar{U}_{r}$ can be expressed as

$$
\begin{aligned}
\bar{U}_{r} & =\int_{-\infty}^{+\infty} \int_{-\infty}^{+\infty} \int_{-\infty}^{+\infty} \int_{-\infty}^{+\infty} \int_{-\infty}^{+\infty} \int_{-\infty}^{+\infty} U_{k} f\left(\vec{U}_{i}, \vec{U}_{j}\right) \mathrm{d} U_{i, x} \mathrm{~d} U_{i, y} \mathrm{~d} U_{i, z} \mathrm{~d} U_{j, x} \mathrm{~d} U_{j, y} \mathrm{~d} U_{j, z} \\
& =\sqrt{8 / \pi} \sqrt{\sigma_{U_{i}}^{2}+\sigma_{U_{j}}^{2}}
\end{aligned}
$$

The variances $\sigma_{U_{i}}^{2}$ and $\sigma_{U_{i}}^{2}$ appearing in eqn (26) needs to be given. The motion of droplets is mainly driven by eddies in turbulent flows, the mean square velocity of droplets, $U_{i}^{2}$, could be estimated from the mean kinetic energy of droplets obtained from eddies, $e_{d_{i}}(\lambda)$. In addition, Han et al. [17] argued that $\bar{e}_{d_{i}}(\lambda)$ could be estimated from the mean available kinetic energy of eddies, $\bar{e}_{\text {available }}(\lambda)$. Hence, $U_{i}^{2}$ could be given as

$$
\overline{U_{i}^{2}}=\bar{e}_{d_{i}}(\lambda) / m_{d_{i}}=\bar{e}_{\text {available }}(\lambda) / m_{d_{i}}=12 \bar{e}_{\text {available }}(\lambda) /\left(\pi \rho_{d} d_{i}^{3}\right)
$$

Note that, turbulent eddy $\lambda_{\text {new }}$ is formed by turbulent eddy $\lambda$ in single-eddy mechanism. The average kinetic energy of turbulent eddies of size $\lambda_{\text {new }}$ can be roughly estimated by $\bar{e}(\lambda)-\bar{e}_{\text {avaiable }}(\lambda)$, then the available kinetic energy of turbulent eddies of size $\lambda_{\text {new }}$ could be easily obtained by using the expression proposed by Han et al. [17].

Since droplet velocity obeys Gaussian distribution, $\overline{U_{i}^{2}}$ can be calculated by eqn (28).

$$
\overline{U_{i}^{2}}=\int_{-\infty}^{+\infty} \int_{-\infty}^{+\infty} \int_{-\infty}^{+\infty} \frac{U_{i}^{2}}{\left(2 \pi \sigma_{U_{i}}^{2}\right)^{3 / 2}} \exp \left[-U_{i}^{2} /\left(2 \sigma_{U_{i}}^{2}\right)\right] \mathrm{d} U_{i, x} \mathrm{~d} U_{i, y} \mathrm{~d} U_{i, z}=3 \sigma_{U_{i}}^{2}
$$

Hence, the variances could be determined by eqns (27) and (28). That is

$$
\sigma_{U_{i}}=\chi_{\sigma}\left[4 \bar{e}_{\text {available }}(\lambda) /\left(\pi \rho_{d} d_{i}^{3}\right)\right]^{1 / 2}
$$

Mean available kinetic energy is obtained by assuming a sinusoidal velocity distribution inside an eddy and the velocity PDF closely follows Gaussian distribution only valid in homogeneous isotropic turbulence. $\chi_{\sigma}$ in eqn (29) is used to calibrate these assumptions.

\subsubsection{Mean Collision Free Path}

The mean collision free path could be taken as the length of the path divided by the number of collisions during a time interval. Following the derivation process of $\Omega\left(d_{i}, d_{j}\right)$, the number 
of collisions of droplet $d_{i}$ with the set of droplets $d_{j}$ per unit time could be expressed as $\pi R_{i j}^{2} \bar{U}_{r} n_{j}$. During a specific time interval $\mathrm{d} t$, the mean distance travelled by a moving droplet between successive collisions could be expressed as $\bar{U}_{r} \mathrm{~d} t$. Then mean collision free path between two droplets of sizes $d_{i}$ and $d_{j}$ could be determined as

$$
L_{d_{j} \rightarrow d_{i}}=\bar{U}_{r} \cdot \mathrm{d} t /\left(\pi R_{i j}^{2} \bar{U}_{r} n_{j} \cdot \mathrm{d} t\right)=1 /\left(\pi R_{i j}^{2} n_{j}\right)
$$

$L_{\lambda \rightarrow d}$ could be also determined base on eqn (2). To be noted that, $L_{\lambda \rightarrow d}^{*}$ in eqn (2) is used to modify the influence of $\tau_{e}$ on the mean collision free path, and $L_{\lambda \rightarrow d}^{*}$ needs to be replaced by $L_{\lambda \rightarrow d}$ when eqn (2) is applied to estimate $L_{\lambda \rightarrow d^{*}}$ Similar to eqn (10), the number of collision of droplet $d$ with the set of eddies $\lambda$ per unit time could be expressed as $0.5 \pi R_{\lambda, d}^{2} \bar{u}_{\lambda} N_{\lambda}$. The number $N_{\lambda}$ of eddy $\lambda$ per unit physical space volume could be roughly estimated by the product of the size of eddies and its number density of sizes between $\lambda$ and $\lambda+\mathrm{d} \lambda$. Following eqn (30), finally the expression of $L_{\lambda \rightarrow d}$ could be estimated by eqn (31).

$$
L_{\lambda \rightarrow d}=\left(\overline{u_{\lambda}} \cdot \mathrm{d} t\right) /\left[\left(0.5 \pi R_{\lambda, d}^{2} \overline{u_{\lambda}} N_{\lambda} \cdot \mathrm{d} t\right)\right]=8 /\left[\pi(\lambda+d)^{2} \lambda n_{\lambda}\right]
$$

The expressions of $L_{\lambda \rightarrow d_{i}}, L_{\lambda_{i} \rightarrow d_{i}}, L_{\lambda_{j} \rightarrow d_{j}}$ and $L_{\lambda_{\text {new }} \rightarrow d_{j}}$ are similar to eqn (31). Note that in single-eddy mechanism, since the eddy $\lambda_{\text {new }}$ results from the volume dilatation of the eddy $\lambda$, the number density of eddy $\lambda_{\text {new }}$ should be estimated by $P\left(\lambda, d_{i}\right) n_{\lambda}$.

\section{RESULTS AND DISCUSIONS}

\subsection{Effect of Integration Limit}

The effect of integration limits of $\lambda_{\min }$ and $\lambda_{\max }$ in eqns (15)-(24) is tested in this section. Since eddies in the entire energy spectrum contribute to the total coalescence rate, $\lambda_{\text {min }}$ could be taken as $\eta$ for covering the contribution of eddies in the dissipation range. The specific total coalescence rate (i.e. $\left.C_{\text {tot }}\left(d_{i}, d_{j}\right) /\left(n_{i} n_{j}\right)\right)$ predicted by eqn (24) is shown in Fig. 2(a). The calculation results show that the main contribution to coalescence rates of small droplets (i.e. $d_{i}<0.1 \mathrm{~mm}$ ) comes from large eddies. Furthermore, the contribution of large eddies to coalescence rate decreases with increasing droplet size. Especially, eddies with size larger than $20 d_{e q}$ have a little effect on total coalescence rate, thus $\lambda_{\text {max }}$ can be taken as $20 d_{e q}$.
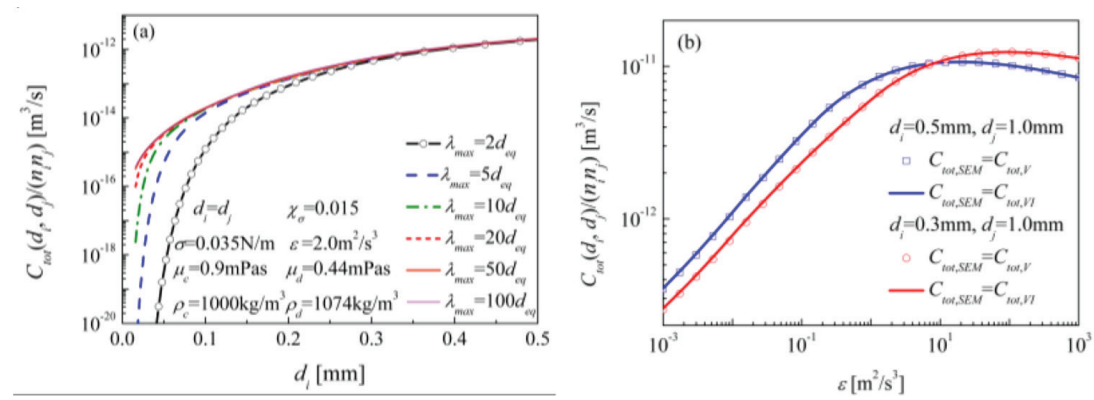

Figure 2: (a) Effect of integration limit on the total coalescence rate; (b) Effects of case V and case VI on the total coalescence rate. 
Furthermore, it could be find that the specific total coalescence rate predicted by eqn (24) adopting $C_{t o t, S E M}=C_{t o t, V}$ and $C_{t o t, S E M}=C_{t o t, V I}$ are close in values each other, please see Fig. 2(b). It indicates that the cases of $\mathrm{V}$ and VI have similar contribution to the total coalescence rate. Hence, the expression of $C_{t o t, S E M}=C_{t o t, V}$ is adopted in this work to predict the total coalescence rate.

\subsection{Comparison of Different Collision Rate Models}

If the coalescence efficiency term is not considered in eqn (24), the total collision rate $\omega_{t o t}\left(d_{i}\right.$, $d_{j}$ ) of two droplets could be obtained. The comparison of the predicted results by different collision rate models is shown in Fig. 3(a). As a whole, the specific total collision rate (i.e. $\left.\omega_{t o t}\left(d_{i}, d_{j}\right) /\left(n_{i} n_{j}\right)\right)$ predicted by these models exhibits a monotonous tendency with droplet size. The proposed model predicts the smallest value of specific total collision rate. However, the specific total collision rate predicted by other models is several orders of magnitude larger than that predicted by the proposed model. It is because the interactions between eddies and droplets were not considered in previous models. It also indicates that the interactions between eddies and droplets have a significant influence on the collision rate between two droplets. Note that, the number density of droplets should be given before the model of Wang et al. [3] can be used. It is assumed that the number density of droplets obeys an exponential distribution, that is $n=4 n_{0} v \exp \left(-2 v / v_{m}\right) / v_{m}{ }^{2}$. Here, $v$ is the volume of droplet, $v_{m}$ denotes the mean volume in droplet size space, and $n_{0}$ is the total number of droplet.

\subsection{Comparison of Different Coalescence Rate Models}

As shown in Fig. 3(b), the coalescence rates predicted by different models exhibit different trends when turbulent energy dissipation rate $\varepsilon$ increases. The model of Das [16] predicts that increasing $\varepsilon$ increases the coalescence rate. The coalescence rate predicted by the model of Lehr et al. [2] increases for small $\varepsilon$, but the coalescence rate is nearly independent of $\varepsilon$ for large $\varepsilon$. For the other coalescence rate models in Fig. 3(b), although there is quantitative difference among these models, it could be found that the coalescence rates predicted by these models are increased firstly and then decreased as increasing $\varepsilon$. Due to lack of experimental
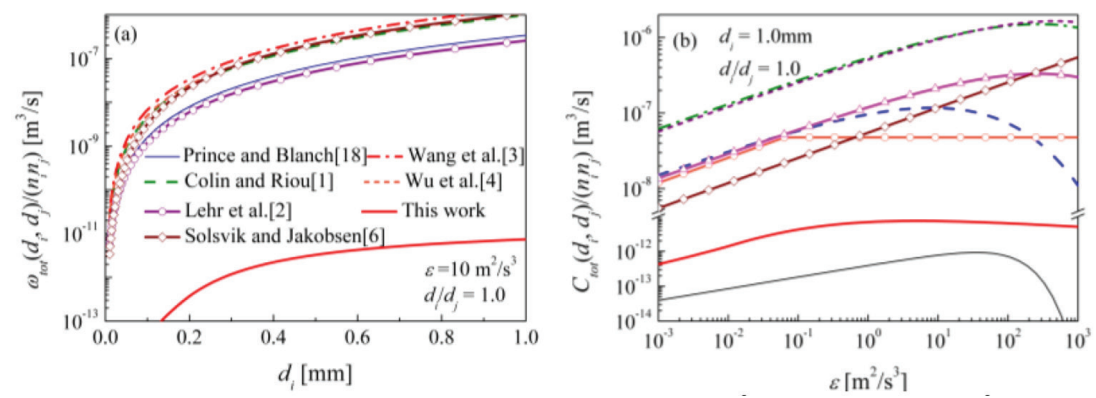

Figure 3: Comparison of different models $\left(\rho_{c}=1000 \mathrm{~kg} / \mathrm{m}^{3}, \rho_{d}=870 \mathrm{~kg} / \mathrm{m}^{3}, \mu_{c}=1.0 \mathrm{mPa} \cdot \mathrm{s}\right.$, $\mu_{d}=0.44 \mathrm{mPa} \cdot \mathrm{s}, \sigma=0.058 \mathrm{~N} / \mathrm{m}, \chi_{\sigma}=0.015, \alpha_{d}=0.05, v_{m}=4.02 \times 10^{-11} \mathrm{~m}^{3}, n_{0}$ $=5 \times 10^{10}$ ). (a) collision rate; (b) coalescence rate, - Coulaloglou and Tavlarides [7]; - - Prince and Blanch [18]; - - Luo [10]; -... Kamp et al. [8]; - - Lehr et al. [2]; -- Wang et al. [3]; - - Das [16]; — this work. 
data, it is difficult to determine which trend is correct. More experiments are needed to fully validate the model and guide further model improvement.

\subsection{Experimental Validation}

One method to validate the coalescence rate model is to couple the model with population balance model to predict the evolution of droplet size distribution. The pure coalescence experiment in stirred tank with six-bladed Rushton impeller was performed by Tobin et al. [19] through dropping the impeller speed from 600rpm to 200rpm suddenly, and the cumulative size distributions of water heptane- $\mathrm{CCl}_{4}$ system $\left(\alpha_{d}=5 \%\right)$ were measured by them. The experimental data of Tobin et al. [19] will be applied to test the proposed model. In the experiment of Tobin et al. [20], the mean turbulent energy dissipation rate $\varepsilon_{m}$ roughly equals to $0.013 \mathrm{~m}^{2} / \mathrm{s}^{3}$ according to Tsouris and Tavlarides [20].

The predicted cumulative volume fraction distributions are compared with the experimental data of Tobin et al. [19], please see Fig. 4. The experimental data at $1 \mathrm{~min}$ is used as the initial condition to solve population balance equation in Fig. 4. Meanwhile, the sizes of droplets are divided into 61 size groups for the experimental system. In addition, the built-in function quad2d in the MATLAB is used here to calculate the double integrals in the coalescence rate model. Note that, the value of $\chi_{\sigma}$ is specified as 0.015 . Although the value of $\chi_{\sigma}$ is small, it may be reasonable since the contribution of eddies of various sizes to the coalescence of droplets of given size is considered in the proposed model. As presented in Fig. 4(a),
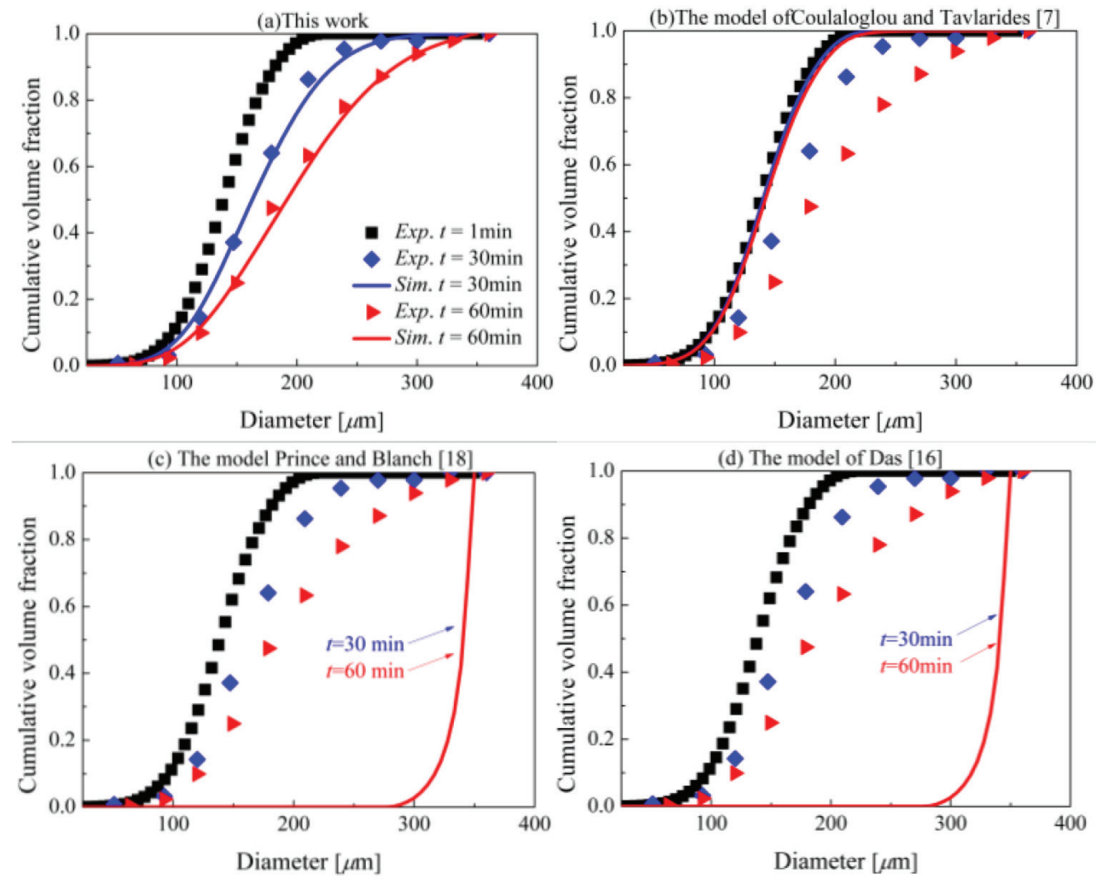

Figure 4: Comparison of experimental data of Tobin et al. [19] with size distributions predicted by proposed model and other models $\left(\varepsilon_{m} \approx 0.013 \mathrm{~m}^{2} / \mathrm{s}^{3}, \rho_{c}=1000 \mathrm{~kg} / \mathrm{m}^{3}\right.$, $\left.\rho_{d}=1000 \mathrm{~kg} / \mathrm{m}^{3}, \mu_{c}=0.90 \mathrm{mPa} \cdot \mathrm{s}, \mu_{d}=0.44 \mathrm{mPa} \cdot \mathrm{s}, \sigma=0.021 \mathrm{~N} / \mathrm{m}, \chi_{\sigma}=0.015\right)$. 
it is observed that the size distributions predicted by the proposed model are in an agreement with experimental data. Moreover, the model of Coulaloglou and Tavlarides [7] seems to underestimate the size distributions, while the model of Das [16] as well as the model of Prince and Blanch [18] extremely overestimates the size distributions. It is because the droplet coalescence rate predicted by these models is extremely larger than or less than that predicted by the proposed model (please see Fig. 3(b)).

Note that, the predicted results at $t=30 \mathrm{~min}$ in Figs. 4(c) and 4(d) nearly coincides with the predicted results at $t=60 \mathrm{~min}$. In the calculation code, the generation rate of droplet of volume larger than the volume of the largest droplet in the experimental system is set to 0 in order to ensure the conservation of volume of droplets in the whole simulation process. If the coalescence rate model of Prince and Blanch [18] (or Das [16]) is employed, the small droplets will rapidly coalescence into large droplets in a short time. When $t=30 \mathrm{~min}$, the sizes of droplets predicted by the model are basically comparable to the size of the largest droplet in the experimental system. Hence, the results predicted by these two models in Figs. 4(c) and 4(d) at $t=60 \mathrm{~min}$ are consistent with that at $t=30 \mathrm{~min}$, respectively.

\section{CONCLUSIONS}

This work mainly focused on the droplet coalescence caused by turbulence. Two different mechanisms between eddies and droplets were proposed, and the mathematical models have been proposed for these two mechanisms. Different from the previous work, the contribution of eddies of various sizes to the coalescence of droplets of given sizes were considered in the framework of entire energy spectrum. This work also considered the influences of life time of eddies and the mean collision free path between eddies and droplets on droplet coalescence.

According to the predicted results of proposed model, it could be found that the life time of eddies and the mean collision free path between eddies and droplets have an important effect on droplet coalescence. Moreover, the predicted results by coupling the proposed model with population balance model showed an agreement with experimental data.

Further experimental studied are urgently needed to fully validate the model and guide the model improvement.

\section{REFERENCES}

[1] Colin, C. \& Riou, X., Turbulence and shear-induced coalescence in gas-liquid pipe flows. 5th International Conference on Multiphase Flow, 2004.

[2] Lehr, F., Millies, M. \& Mewes, D., Bubble-size distributions and flow fields in bubble columns. AIChE J, 48(11), pp. 2426-2443, 2002. https://doi.org/10.1002/aic.690481103

[3] Wang, T.F., Wang, J.F. \& Jin, Y., Theoretical prediction of flow regime transition in bubble columns by the population balance model. Chemical Engineering Science, 60(22), pp. 6199-6209, 2005. https://doi.org/10.1016/j.ces.2005.04.027

[4] Wu, Q., Kim, S., Ishii, M. \& Beus, S., One-group interfacial area transport in vertical bubbly flow. International Journal of Heat and Mass Transfer, 41(8), pp. 1103-1112, 1998. https://doi.org/10.1016/s0017-9310(97)00167-1

[5] Han, L.C., Gong, S., Li, Y., Gao, N., Fu, J., Luo, H. \& Liu, Z., Influence of energy spectrum distribution on drop breakage in turbulent flows. Chemical Engineering Science, 117, pp. 55-70, 2014. https://doi.org/10.1016/j.ces.2014.06.018 
[6] Solsvik, J. \& Jakobsen, H.A., Development of fluid particle breakup and coalescence closure models for the complete energy spectrum of isotropic turbulence. Industrial \& Engineering Chemistry Research, 55(5), pp. 1449-1460, 2016.

https://doi.org/10.1021/acs.iecr.5b04077

[7] Coulaloglou, C. \& Tavlarides, L., Description of interaction processes in agitated liquid-liquid dispersions. Chemical Engineering Science, (11), pp. 1289-1297, 1977. https://doi.org/10.1016/0009-2509(77)85023-9

[8] Kamp, A., Chesters, A., Colin, C. \& Fabre, J., Bubble coalescence in turbulent flows: a mechanistic model for turbulence-induced coalescence applied to microgravity bubbly pipe flow. International Journal of Multiphase Flow, 27(8), pp. 1363-1396, 2001. https://doi.org/10.1016/s0301-9322(01)00010-6

[9] Lee, C.H., Erickson, L.E. \& Glasgow, L.A., Bubble breakup and coalescence in turbulent gas-liquid dispersions. Chemical Engineering Communications, 59(1-6), pp. 65-84, 1987. https://doi.org/10.1080/00986448708911986

[10] Luo, H.A., Coalescence, break-up and liquid circulation in bubble column reactors. Norwegian Institute of Technology, 1993.

[11] Peev, N., Particle collision in amorphous medium at equilibrium. Journal of Research in Physics, 31(1), pp. 69-78, 2007.

[12] Pope, S.B., Turbulent flows, Cambridge University Press, 2000.

[13] Hinze J.O., Turbulence, McGraw-Hill, 1975.

[14] Sawford, B. \& Hunt, J., Effects of turbulence structure, molecular diffusion and source size on scalar fluctuations in homogeneous turbulence. Journal of Fluid Mechanics, 165, pp. 373-400, 1986. https://doi.org/10.1017/s0022112086003142

[15] Luo, H.A. \& Svendsen, H.F., Theoretical model for drop and bubble breakup in turbulent dispersions. AIChE Journal, 42(5), pp. 1225-1233, 1996.

https://doi.org/10.1002/aic.690420505

[16] Das, S.K., Development of a coalescence model due to turbulence for the population balance equation. Chemical Engineering Science, 137, pp. 22-30, 2015. https://doi.org/10.1016/j.ces.2015.04.026

[17] Han, L.C., Luo, H.A. \& Liu, Y.J., A theoretical model for droplet breakup in turbulent dispersions. Chemical Engineering Science, 66(4), pp. 766-776, 2011. https://doi.org/10.1016/j.ces.2010.11.041

[18] Prince, M.J. \& Blanch, H.W., Bubble coalescence and break-up in air-sparged bubble columns. AIChE Journal, 36(10), pp. 1485-1499, 1990. https://doi.org/10.1002/aic.690361004

[19] Tobin, T., Muralidhar, R., Wright, H. \& Ramkrishna, D., Determination of coalescence frequencies in liquid-liquid dispersions: effect of drop size dependence. Chemical Engineering Science, 45(12), pp. 3491-3504, 1990. https://doi.org/10.1016/0009-2509(90)87154-k

[20] Tsouris, C. \& Tavlarides, L., Breakage and coalescence models for drops in turbulent dispersions. AIChE Journal, 40(3), pp. 395-406, 1994.

https://doi.org/10.1002/aic.690400303 